\title{
Sistema de autoconstrucción sismorresistente: características resistentes y proceso constructivo
}

\section{Earthquake resistant self-build system: strength characteristics and constructive process}

B. Orta $^{(*)}$, J. Adell ${ }^{(*)}$, R. Bustamante ${ }^{(*)}$, S. Martínez-Cuevas ${ }^{(* *)}$

RESUMEN

La autoconstrucción con tierra, adobe o ladrillo es muy habitual en los países en vías de desarrollo que están en zonas de alto riesgo sísmico. Ante la necesidad de dar respuesta al problema, aportando sencillez constructiva, hemos trabajado desde hace años en el empleo del Sistema de Albañilería Integral (SAI).

El SAI es un sistema que, combinado con materiales autóctonos, aporta resistencia sísmica y su facilidad de ejecución lo hace adecuado para la autoconstrucción de viviendas antisísmicas.

Este artículo describe el SAI, desarrolla el proceso constructivo aplicado a una vivienda tipo de dos alturas y muestra su comportamiento sismo-resistente mediante los resultados obtenidos en tres campañas de ensayos. La originalidad del sistema radica en el empleo de un solo tipo de armaduras prefabricadas en forma de cercha que se entrelazan entre si en las tres direcciones del espacio permitiendo crear una malla tridimensional.

Palabras clave: vivienda sismorresistente; autoconstrucción; Sistema de Albañilería Integral; materiales autóctonos o reciclados; adobe; ladrillo hueco.

\section{ABSTRACT}

The dwelling construction with soil, adobe or brick without call for technical advice is very typical in countries in process of development that are in areas of high seismic risk. To give an answer to the problem of earthquake building using these materials, providing simplicity of construction, we have worked for years in the use of the Integral Masonry System (IMS).

The IMS is a system that, used combined with native materials, has earthquake resistant properties and its ease of implementation makes it suitable for self-build earthquake-resistant dwellings.

This article describes the IMS, develops the constructive process applied to a type of two storey houses, and shows the seismic behaviour by means of the results obtained in the three test campaigns. The originality of the system lies in the use of a single type of prefabricated trusses, intertwined in the three spatial directions allowing create a dimensional mesh.

Keywords: earthquake resistant construction; Integral Masonry System; native materials; recycled materials; adobe; hollow brick.

(") Universidad Politécnica de Madrid - Escuela Técnica Superior de Arquitectura de Madrid. España.

${ }^{(* *)}$ Universidad Politécnica de Madrid - Escuela Técnica Superior de Ingenieros en Topografía Geodesia y Cartografía.

Persona de contacto/Corresponding author: belen.orta@upm.es (B. Orta)

Cómo citar este artículo/Citation: Orta, B.,Adell, J., Bustamante, R., Martínez-Cuevas, S. (2015). Sistema de autoconstrucción sismorresistente: características resistentes y proceso constructivo. Informes de la Construcción, 68(542): e145, doi: http://dx.doi.org/10.3989/ic.15.082.

Licencia / License: Salvo indicación contraria, todos los contenidos de la edición electrónica de Informes de la Construcción se distribuyen bajo una licencia de uso y distribución Creative Commons Attribution License (CC BY) Spain 3.o. 


\section{INTRODUCCIÓN}

La construcción en tierra, adobe o ladrillo está muy extendida en los países en vías de desarrollo, tanto por su facilidad constructiva como por su coste (6). La experiencia y los ensayos de laboratorio (5) (7) (13) (14) (15) indican que estos materiales no son apropiados para una construcción antisísmica. Se trata de viviendas autoconstruidas sin asesoría técnica. Surge por tanto la necesidad de dar respuesta a las poblaciones de estos países que sufren la acción sísmica tales como los recientes de Pisco (Perú) en Agosto 2007, de Haití en Enero 2010, de Chile en 2010 y 2014, de Van (Turquía) en octubre 2011, o de Nepal en abril de 2015, que por la deficiente resistencia de sus edificaciones que generan más muertes que el sismo en sí (9) (10). Este artículo muestra un sistema constructivo basado en los materiales locales para la reconstrucción de esas áreas devastadas, armándolos con una nueva técnica que asegure la resistencia ante la acción sísmica, denominado Sistema de Albañilería Integral (SAI).

Se han ensayado tres prototipos de vivienda sismorresistente con el SAI, en el marco del convenio de colaboración entre las Universidades ETSAM-UPM Madrid España y PUCP Lima Perú. Se trata de una vivienda cuadrada de 2 plantas, $6 \mathrm{~m}$ de ancho y $6 \mathrm{~m}$ de altura: el primer ensayo a escala $1 / 2$ con relleno de adobe y mortero del mismo barro en las juntas en 2008 (proyecto ALo8-P(i+D)-01); el segundo ensayo a escala $1 / 2$ con relleno de ladrillo hueco y mortero de cemento en 2009 (proyecto ALo9-P(i+D)-02) y el tercer ensayo a escala $1 / 1$ pero con sólo un $1 / 4$ del edificio de ladrillo hueco y mortero de cemento en 2011 (proyecto AL11$\mathrm{P}(\mathrm{i}+\mathrm{D})-\mathrm{O1})$, dado el tamaño de la plataforma de ensayo de la PUCP de sólo $4 \times 4 \mathrm{~m}$

\section{DESCRIPCIÓN DEL SISTEMA DE ALBAÑILERÍA INTEGRAL (SAI)}

El Sistema de Albañilería Integral (SAI) (2) (3) desarrollado en España mediante Patente comercializada por AllWall ${ }^{\circledR}$ Systems, es un sistema constructivo que permite el armado homogéneo de las fábricas tanto vertical como horizontalmente con y sin mortero. El armado se realiza mediante el empleo de las Costilla ${ }^{\circledR}$ Allwall ${ }^{\circledR}$ igualmente patentada en España.

El SAI consiste en un tipo único de armaduras prefabricadas en forma de cercha de $6 \mathrm{~m}$ de longitud que pueden entrelazarse entre sí fácilmente y sin necesidad de conocimientos técnicos, en las tres direcciones del espacio para constituir muros, forjados o cubiertas, relleno de materiales locales como adobe, ladrillo o materiales reciclados facilitando la autoconstrucción. El conjunto tiene comportamiento resistente a sismo como se verá a continuación.

La longitud de las cerchas permite su transporte en camiones estándar y edificar hasta 2 plantas con luces hasta 6 metros de cualquier tipología y material, ya sea de fábrica (material reciclado, adobe, ladrillo, bloque...), elementos de chapa, madera o prefabricados de hormigón.

\subsection{Componentes del Sistema}

Estas armaduras de refuerzo en forma de cercha son una solución de continuidad estructural gracias a su longitud de $6 \mathrm{~m}$ que abarca tanto la longitud total de la vivienda tipo dos plantas de altura. Se prefabrican con dos cantos diferentes $240 \mathrm{~mm}$ y $220 \mathrm{~mm}$ de canto (Figura 1), lo que facilita el montaje y entrelazado. Como se entrelazan tridimensionalmente, las de menor tamaño están diseñadas para que se ajusten por el interior de la de mayor anchura sin apenas holgura para que su sujeción sea más precisa, esta operación de enhebrado se puede realizar a mano. Esta combinación de cerchas se emplea en la construcción de forjados y muros. Las de mayor anchura $(240 \mathrm{~mm})$ serán las costillas verticales de los muros, reservando las más estrechas (220 $\mathrm{mm}$ ) como refuerzo horizontal. El dimensionado corresponde con los cálculos realizados conforme a la normativa sísmica de Perú (16).

Se prefabrican con acero B-500-S con recubrimiento galvanizado para evitar su corrosión.

El material de relleno que da la rigidez final al conjunto puede ser una fábrica de cualquier material autóctono de construcción tradicional (tierra, adobe, ladrillo, bloque de hormigón, etc.) así como materiales reciclados (aquellos aprovechables derivados de la destrucción de construcciones devastadas por un terremoto).

\section{TIPOLOGÍAS DE VIVIENDAS SISMORESISTENTES CON EL SAI PARA PERÚ}

En SismoAdobe 2005 (1) se presentó el diseño de 6 viviendas tipo (Tabla 1). basadas en las medidas estándar de la Costilla a prefabricar del Sistema AllWall de 5,85 m, para transportarse con facilidad en la caja de un camión pequeño, con una ligereza de $8 \mathrm{~kg} /$ pieza aproximadamente, las viviendas sismorresistentes que aquí se proponen ofrecen diversas tipologías y geometrías para elegir en función de las necesidades del usuario final de la vivienda y del contexto social son viviendas de 1 y de 2 plantas.
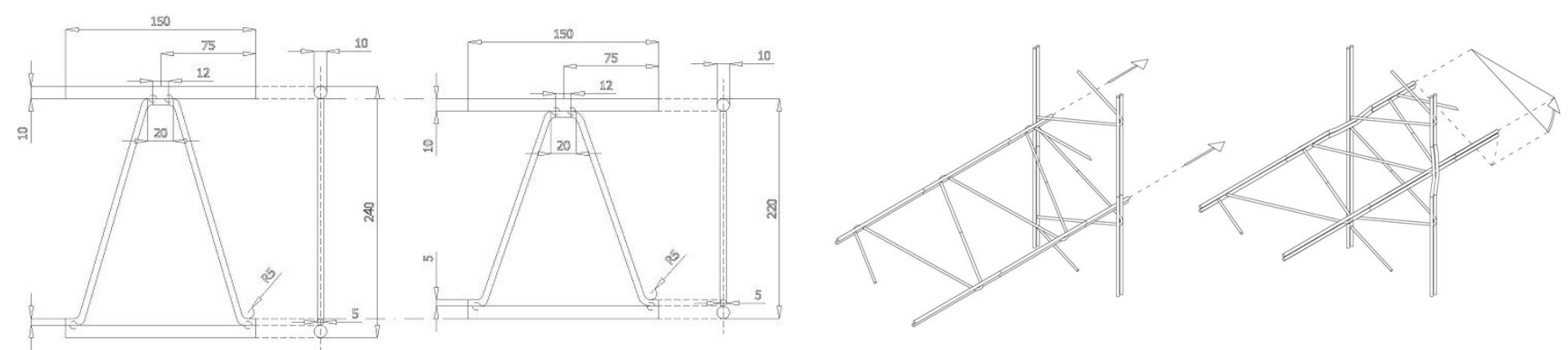

Figura 1. Detalles de cerchas AllWall ${ }^{\circledR}$ para las paredes y forjados de viviendas resistentes al seísmo y entrelazado manual. 
Tabla 1. Prototipo de viviendas.

\begin{tabular}{|c|c|c|c|c|}
\hline Tipo & Dimensiones & Superficie construida & Superficie de planta & Dormitorios \\
\hline 1 & $5,10 \times 6,00 \mathrm{~m}$ & $30,6 \mathrm{~m}^{2}$ & $24,3 \mathrm{~m}^{2}$ & 1 \\
\hline 2 & $6,00 \times 6,00 \mathrm{~m}$ & $36,0 \mathrm{~m}^{2}$ & $29,2 \mathrm{~m}^{2}$ & 1 \\
\hline 3 & $2(5,10 \times 6,00 \mathrm{~m})$ & $61,2 \mathrm{~m}^{2}$ & $51,3 \mathrm{~m}^{2}$ & 2 \\
\hline 4 & $2(5,10 \times 6,00 \mathrm{~m})$ & $61,2 \mathrm{~m}^{2}$ & $51,3 \mathrm{~m}^{2}$ & 2 \\
\hline 5 & $2(6,00 \times 6,00 \mathrm{~m})$ & $72,0 \mathrm{~m}^{2}$ & $58,4 \mathrm{~m}^{2}$ & 3 \\
\hline 6 & $2[2(5,10 \times 6,00 \mathrm{~m})]$ & $122,4 \mathrm{~m}^{2}$ & $102,6 \mathrm{~m}^{2}$ & 4 \\
\hline
\end{tabular}

\section{PROCESO DE AUTOCONSTRUCCIÓN DE LA VIVIENDA N. ${ }^{\circ}$ CON EL «SAI»}

Se escoge la Vivienda Tipo 5 de planta cuadrada de $6 \mathrm{~m}$ de lado y 2 plantas de altura de $6 \mathrm{~m}$ de alto en total. A continuación, se expone el proceso constructivo de este modelo.

En la Figura 2 se presenta la modulación en planta y en sección de la vivienda tipo. Las cerchas se separan en planta $90 \mathrm{~cm}$ lo que permitirá la distribución de huecos en la fachada o la ejecución de huecos de escalera en la misma modulación. En alzado la modulación aumenta para permitir una mayor altura de huecos, de esta manera no es necesario dintel en los huecos dado que llegan hasta el forjado superior.

El proceso comienza por la ejecución del cimiento, no se describe en detalle pues depende de las características geotécnicas del suelo.

Se parte de una base nivelada sobre la que se replantean de las cuatro esquinas con un perfil de acero galvanizado en $\mathrm{L}$ dispuesto en vertical. Se mantendrán durante todo el proceso para facilitar el replanteo en altura de las cerchas y como refuerzo de las esquinas ante el sismo.

Con las cuatro esquinas se dispone la primera armadura de las fachadas en horizontal a modo de tendel, con mortero que sirve para la modulación del forjado de planta baja. De realizarse un forjado en planta baja en vez de solera (Figura 4 izquierda), se realizará la construcción que se explica en el siguiente apartado.

\subsection{Forjados}

Los forjados se realizan entrecruzando las cerchas ya descritas, al tener una diferencia de cantos de $2 \mathrm{~cm}$ se mete una dentro de la otra, quedando ajustado. El relleno se puede hacer con bañeras, aquí se propone construirlas a base dos filas del mismo ladrillo hueco que se utilizará para las fachadas con lo que se mejora la resistencia al fuego del forjado. Las dos filas de ladrillo alcanzan un canto de $21 \mathrm{~cm}$, disponiendo los ladrillos según se indica en la Figura 3 (izquierda). Una capa de compresión de hormigón, del orden de 4,5 cm hasta alcanzar los $25,5 \mathrm{~cm}$ de canto total, superando los $24 \mathrm{~cm}$ de

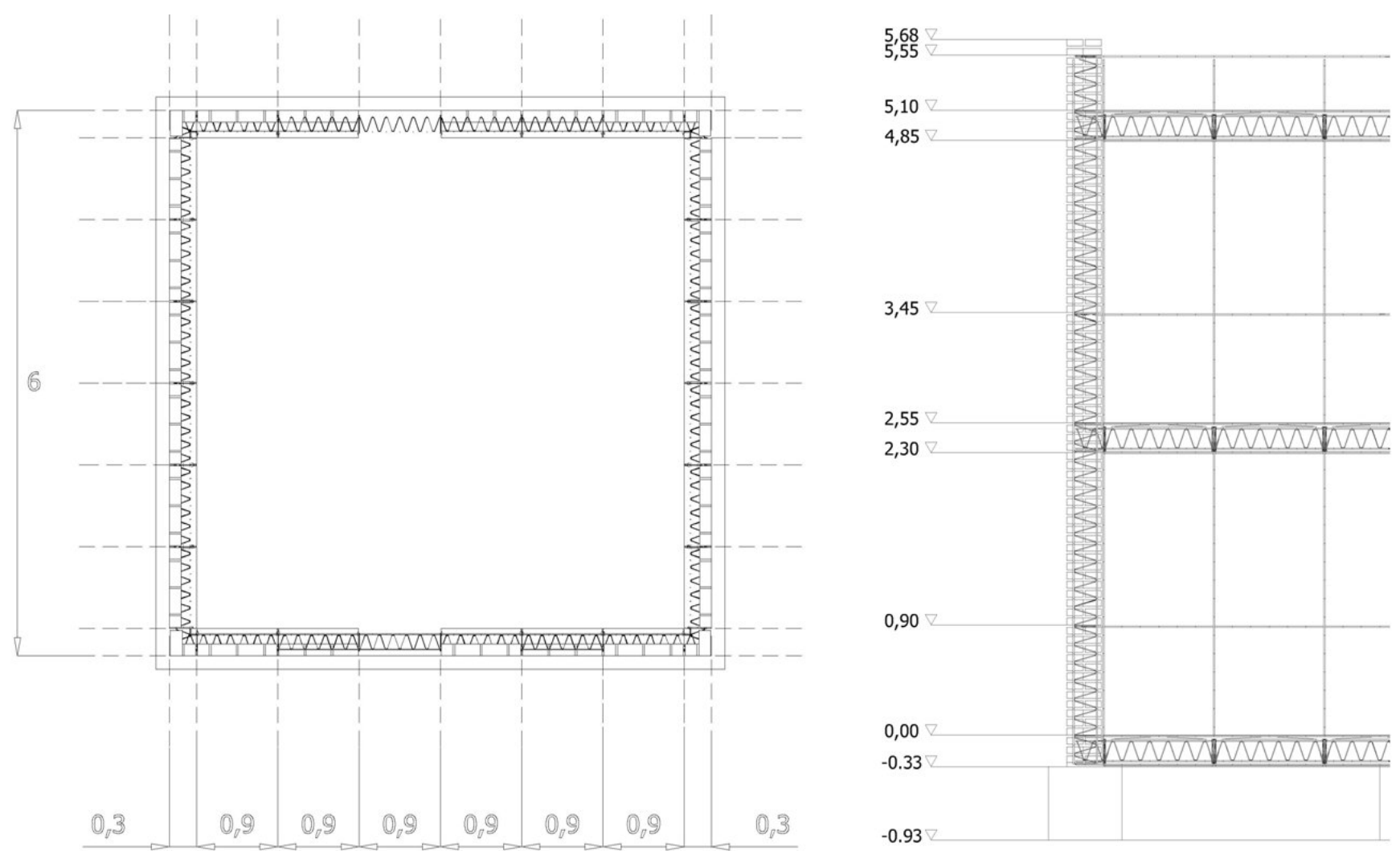

Figura 2. Modulación horizontal y vertical de la vivienda n. ${ }^{0} 5$. 

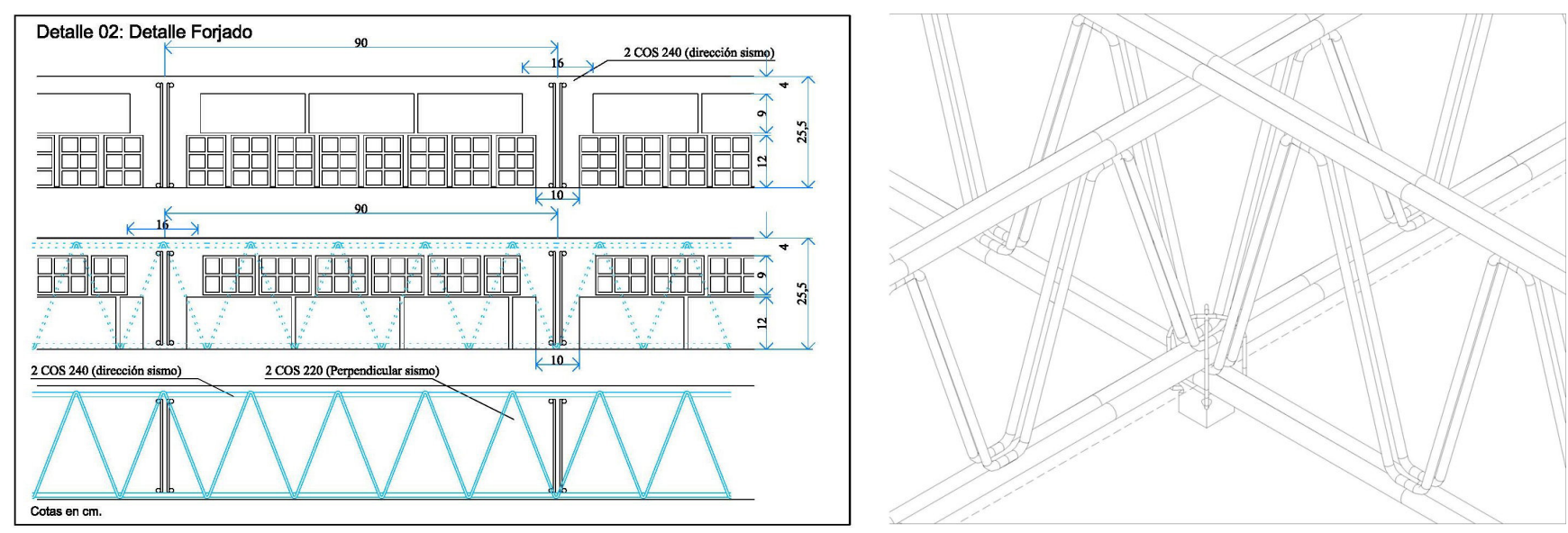

Figura 3. Detalle del forjado construido en el prototipo de ladrillo a escala real. Entrelazado manual de las cerchas en forjado con separador.

canto de las cerchas; también puede utilizarse como relleno piezas de hormigón prefabricado. El funcionamiento es similar al de un forjado reticular con bovedillas cerámicas e intereje $90 \mathrm{~cm}$ con nervios de $10 \mathrm{~cm}$ de ancho.

Los forjados de planta primera y cubierta plana deberán realizarse con protección frente a incendios por medio de unos separadores diseñados por el sistema que garantizan el recubrimiento (Figura 3 derecha).

\subsection{Forjado de planta baja y arranque muros perimetrales}

Tras la colocación de los elementos de forjado de planta baja y antes de verter el hormigón de la capa de compresión hay que colocar las costillas verticales de las fachadas (Figura 4 izquierda) para que el encuentro entre muro y forjado se haga solidario con el hormigón. Las costillas son del total de la altura de la vivienda, $6 \mathrm{~m}$.

Una vez fraguada la capa de compresión de la planta baja las cerchas verticales estarán fijas y se puede proseguir colocando las cerchas horizontales de los muros de fachada, soldadas a los angulares en la esquina. Posteriormente se rellenan los muros de fachada hasta la cota 2,30 m justo por debajo del forjado de planta primera dejando los huecos de puertas y ventanas hasta el techo, sin cargadero propio.

\subsection{Muros de fachada}

Los muros de las fachadas se construyen con las mismas cerchas entrelazadas, en vertical se disponen las cerchas de $24 \mathrm{~cm}$ de canto y en horizontal las de $22 \mathrm{~cm}$ de canto (Figura 5). El relleno se hace con dos hojas de ladrillo de $9 \mathrm{~cm}$ de espesor cada hoja y una cámara de aire intermedia. Las cerchas no quedan vistas y el espesor total del muro es de $26 \mathrm{~cm}$. Al interior de la vivienda se puede enfoscar o dar el acabado que se desee. Las dos hojas quedan enlazadas por las cerchas que hacen de llaves.

\section{ENSAYOS DE VALIDEZ DE LOS PROTOTIPOS SISMORRESISTENTE, REALIZADOS EN LIMA (PERÚ)}

\subsection{Descripción de los Prototipos Ensayados}

Para validar esta solución y su aplicación a la construcción de viviendas resistentes a sismo se procede a realizar tres campañas de ensayos en el Laboratorio de Estructuras Antisísmicas de la PUCP. Dado que ya se poseen resultados de ensayos de los materiales de relleno de manera independiente y a que las cerchas están homologadas en Europa, se ensaya el conjunto de la vivienda.

Los ensayos se realizan con tres prototipos, los dos primeros de la vivienda completa $6 \mathrm{~m} \times 6 \mathrm{~m} \times 6 \mathrm{~m}$; debido al tamaño
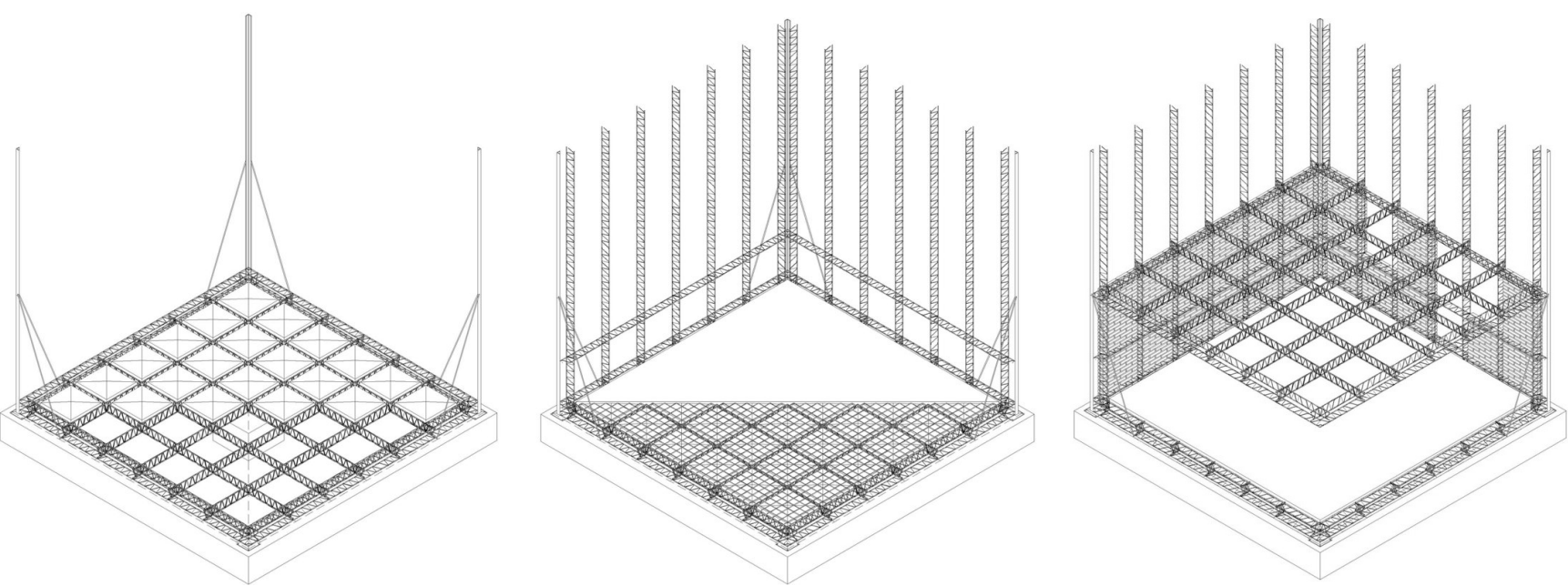

Figura 4. Esquema de ejecución del forjado de planta baja y de las fachadas. 


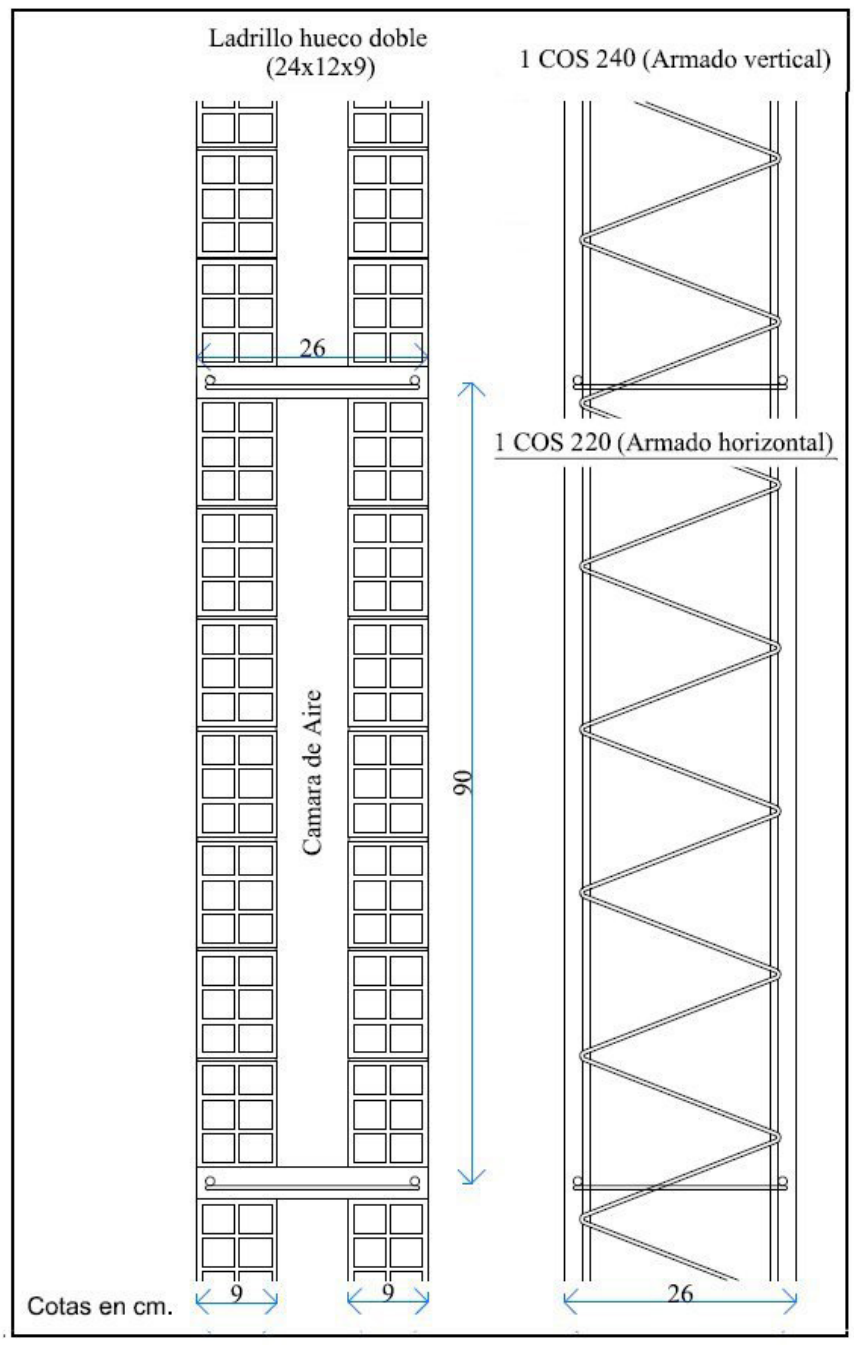

Figura 5. Detalle del muro, sección por el ladrillo y por las cerchas.

de la mesa sísmica se construye a escala mitad por lo que los prototipos mide $3 \mathrm{~m} \times 3 \mathrm{~m} \times 3 \mathrm{~m}$. El primero de ellos es de adobe, con mortero de adobe en las juntas (Figura 6 izquierda), y el segundo de ladrillo hueco con mortero de cemento (Figura 6 derecha). Se escala adecuadamente la sección de armado y el grueso de los muros.
En el tercer prototipo se continúa con el ladrillo hueco utilizando mortero de cemento como material de agarre. Se ensaya a escala real, en esta ocasión se construye 1/4 de vivienda, con lo que se verifican los detalles de unión y la altura total de la estructura. Para simular el comportamiento de la estructura completa, en los ejes de simetría se plantea una estructura metálica que simule la rigidez del resto del edificio que no se construye y así el comportamiento del prototipo sea semejante al de la estructura completa. Además debido a la asimetría del prototipo, para evitar una asimetría en el peso y poder ensayarlo en la mesa sísmica, se regularizó el peso por medio de la ejecución de tabiques no estructurales, separados de los muros para evitar aportar rigidez al conjunto en el borde del prototipo.

En los prototipos 1 y 2 a escala $1 / 2$, se enfoscan dos de las 4 fachadas. Al tratarse de cerchas continuas de $6 \mathrm{~m}$ de longitud, la falta de aparejo entre los distintos recuadros, no supone un debilitamiento de la estructura del Sistema de Albañilería Integral.

El peso de los forjados, no construidos, y sus sobrecarga se sustituye por el peso de sacos de arena aplicados en cada nivel.

Se puede obtener más información sobre los prototipos 1 y 2 en (11) (12) donde se describen con mayor detalle.

El prototipo 3 a escala 1/1 y tamaño de $3 \times 3$ en planta y $6 \mathrm{~m}$ de altura representa $1 / 4$ de la vivienda. Los muros inicialmente eran de dos hojas de medio pie $(12,5 \mathrm{~cm})$ entrelazados con las cerchas a modo de llaves. Se decidió construir los muros de mitad de grueso y añadirlo como contrapeso para equilibrar el peso en la mesa de ensayos. Los muros del prototipo son de una sola hoja de 1/2 pie al exterior del muro. Esto aporta menor resistencia a las fachadas y el mismo peso total del conjunto, por lo que la situación es más desfavorable que la real. En este caso si se construye el forjado con bañeras del mismo ladrillo empleado en las fachadas, en dos filas, según el detalle de la Figura 3. El resto de cargas muertas y sobrecargas se aplica por medio de sacos de arena sobre el forjado (Figura 7).

En la construcción de los tres prototipos el sistema constructivo ha resultado de fácil puesta en práctica, a pesar de ser la primera utilización del sistema en el país, los operarios asimilaron sin mayor dificultad el procedimiento.
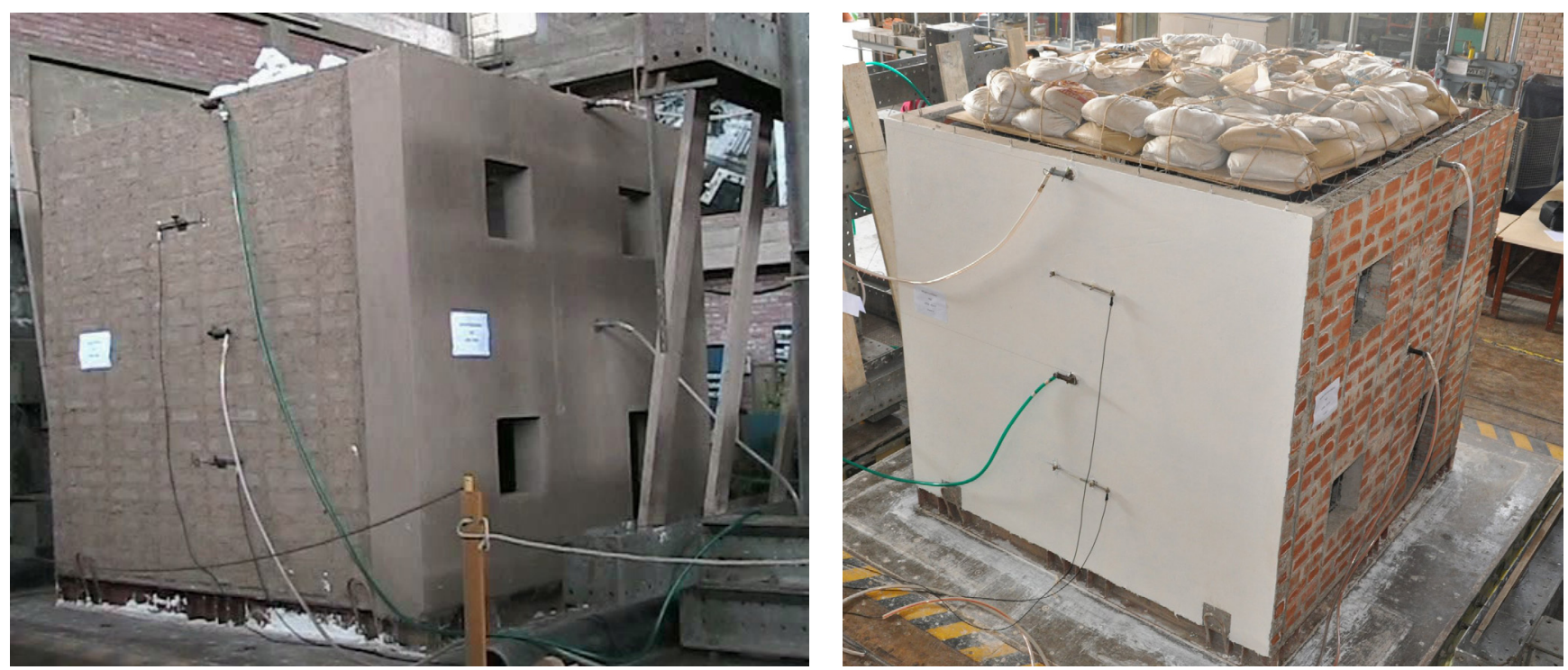

Figura 6. Ensayo de prototipos 1 y 2 con el SAI combinado con adobe o ladrillo hueco. 

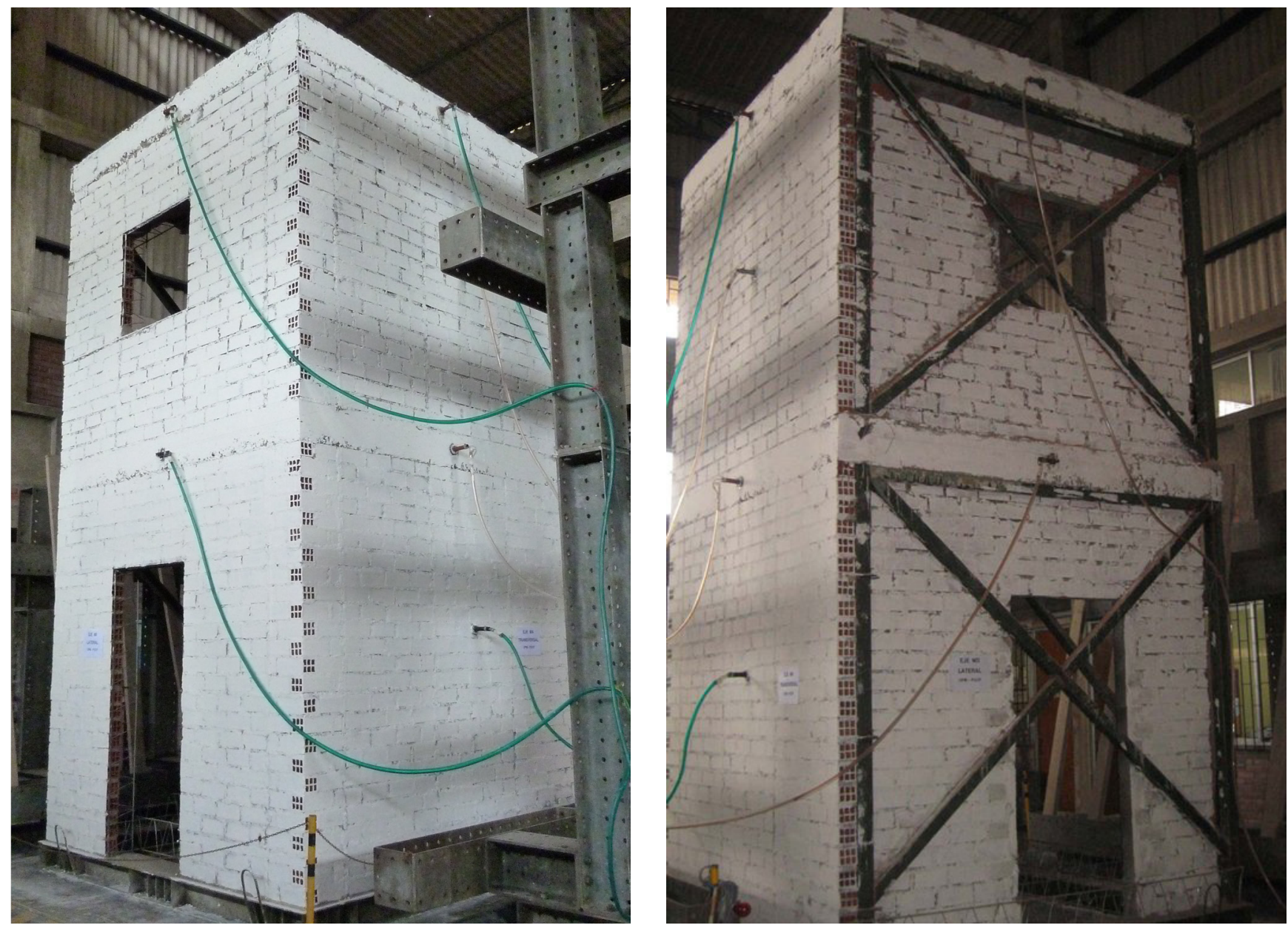

Figura 7. Prototipo 3 terminado, construido con SAI y ladrillo hueco, 1/4 de vivienda a escala real y estructura metálica en los ejes de simetría para simular la rigidez de una vivienda completa.

\subsection{Programa experimental}

Los ensayos se realiza en la mesa sísmica del Laboratorio de Estructuras antisísmicas del Departamento de Ingeniería de la Pontificia Universidad Católica del Perú, que es la entidad más representativa dentro del país. La mesa tiene un solo grado de libertad (de los seis de un sismo real), el desplazamiento en un sentido. Los prototipos se disponen de manera que las fachadas con huecos (puerta y ventanas) quedan perpendiculares al sentido del movimiento y las fachadas ciegas en paralelo.

El movimiento (señal de comando) que se aplica a la plataforma del simulador proviene de la componente longitudinal $\left(\mathrm{N}^{\circ} \mathrm{O}\right.$ ) de la aceleración medida en Lima del Terremoto de Ancash del 31 de mayo de 1970 (Figura 11). El registro original, obtenido en un acelerógrafo analógico, fue procesado por el United States Geological Survey (USGS) para obtener una aceleración digital corregida, que sirvió de base para generar la señal de comando denominada «mayo70» (el estándar en el citado Laboratorio). Esta es la señal de comendo utilizada en el Prototipo 3 a escala real. En los prototipos 1 y 2, debido a su escalado, se utilizó una versión comprimida en el tiempo de esta señal («mayo7oco») modificando sus frecuencias fundamentales en función de las de la estructura escalada. De esta manera se pasa de 100 puntos por segundo de la señal original a 133,3 puntos por segundo en la señal modificada, de acuerdo al factor de escala físico (1/2) del prototipo. Como consecuencia de esta operación, para un desplazamiento de- terminado, la señal comprimida («mayo7oco» equivalente a $130 \mathrm{~mm}$ de desplazamiento) produce aceleraciones mayores que la señal original.

El ensayo dinámico se realizó en una secuencia de movimientos (fases) de amplitud creciente, con la misma señal de comando. Cada fase se define por el desplazamiento máximo absoluto aplicado. Inicialmente se programaron tres primeras fases, en orden creciente de intensidad, correspondientes a un sismo frecuente (leve), uno ocasional (moderado) y uno raro (severo). En el caso de «mayo7oco», esos movimientos corresponden a desplazamientos máximos de $20 \mathrm{~mm}, 50 \mathrm{~mm}$ y $80 \mathrm{~mm}$, respectivamente. Finalmente se someten los prototipos a la amplitud máxima de $130 \mathrm{~mm}$ (capacidad máxima del simulador). Antes del ensayo y después de cada fase se somete a la estructura a una vibración libre, lo que permite conocer su periodo natural de vibración y su variación con el ensayo.

\section{5•3. Resultados y análisis de los ensayos}

Los vídeos de los ensayos muestran un comportamiento sismorresistente (17) (18) de los prototipos. El prototipo 1, de adobe a escala mitad, se agrieta significativamente sin perder la estabilidad (Figura 8). Las grietas se producen marcando las armaduras, más gruesas cerca de la base, como cabía esperar. Los muros perpendiculares a la acción sísmica, son los que menos se agrietan. El periodo natural de la estructura varía poco durante el ensayo y está entorno a T = 0,14 s. 

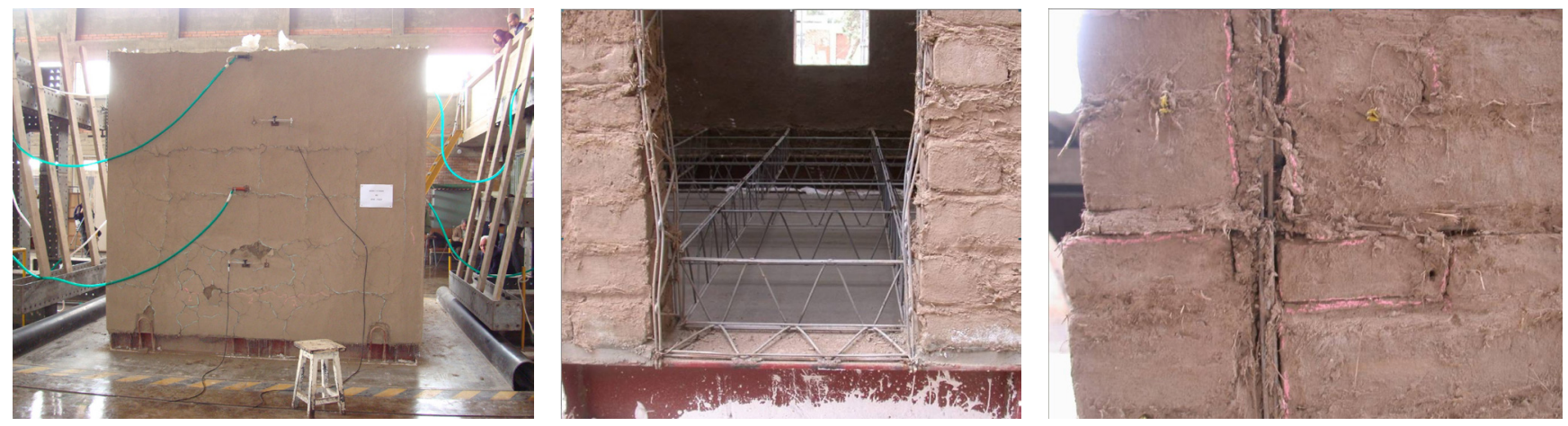

Figura 8. Prototipo 1, de adobe, después del ensayo. Vista general, puerta y una esquina.

En el prototipo 2, de ladrillo a escala mitad, se observan pequeñas fisuras en las piezas de ladrillo y deformaciones del entramado de los forjados y a penas se perciben fisuras generales (Figura 9), sin embargo, los instrumentos de medición si indican fisuras del orden de $0,1 \mathrm{~mm}$. El periodo natural de la estructura varía poco a lo largo del proceso del ensayo y está entorno a $\mathrm{T}=0,13 \mathrm{~s}$.

Los valores de periodos naturales de la estructura de los prototipos 1 y 2 hacen pensar que tienen un comportamiento sísmico intermedio entre una vivienda de 1 y de 2 plantas, esto puede ser debido a la no ejecución completa del forjado intermedio, de ahí la necesidad de ensayar un prototipo completo, el prototipo 3 .

Los puntos débiles del prototipo 3 son las esquinas y los huecos del muro, en la Figura 10 izquierda se observa la fisura junto al hueco de la puerta, incluso las cerchas verticales se despegan del relleno del muro. Se observa la deformación de las barras que tras sufrir una fuerte compresión no han recuperado su rectitud, se trata de la cara MI en la dirección del movimiento. En la cara MD se cae el muro de la planta superior en el cuarto ensayo (Figura 10 centro). Cabía esperar este comportamiento debido a que no estaba atado al resto de la estructura y su misión era simplemente la de aportar un peso simétrico a la mesa de ensayos y sin función estructural alguna. El periodo natural de la estructura varía ligeramente a lo largo del proceso del ensayo, la vibración libre después de las fases da un periodo natural de $\mathrm{T}=0,2 \mathrm{~s}$. Se observa como corresponde con el periodo natural de un edificio de dos plantas.

Los valores máximos de los instrumentos de medición de los prototipos 1 y 2 se pueden consultar en (12), los del prototipo 3 se detallan en la Tabla 2, donde MI y MD son los muros izquierdo y derecho respectivamente en la dirección del mo-

Tabla 2. Valores máximos de los instrumentos de medición (Ao-A8 que miden aceleraciones) en el prototipo 3 .

\begin{tabular}{|c|c|c|c|c|}
\hline & Fase 1 & Fase 2 & Fase 3 & Fase 4 \\
\hline En la base: Ao (g) & 0,28 & 0,8 & 1,5 & 1,2 \\
\hline MI: forjado de planta $1^{\mathrm{a}}$ : A1 (g) & 0,32 & 0,9 & 1,6 & 1,8 \\
\hline MI: forjado de cubierta: A2 (g) & 0,45 & 1,1 & 2,4 & 2,4 \\
\hline MD: forjado de planta $1^{\mathrm{a}}: \mathrm{A} 3(\mathrm{~g})$ & 0,4 & 1,1 & 1,7 & 2,4 \\
\hline MD: forjado de cubierta: A4 (g) & 0,46 & 1,4 & 2,6 & 2,8 \\
\hline MA: forjado planta $1^{\mathrm{a}}:$ A5 (g) & 0,37 & 0,9 & 1,6 & 2 \\
\hline MA: forjado de cubiertaA6 (g) & 0,41 & 1,3 & 2,5 & 2,5 \\
\hline MA: entre baja y 1ª : A7 (g) & 0,35 & 1,2 & 2 & 2,6 \\
\hline MA: entre $1^{\mathrm{a}}$ y cubierta: A8 (g) & 0,43 & 1,2 & 2,1 & 2,1 \\
\hline
\end{tabular}
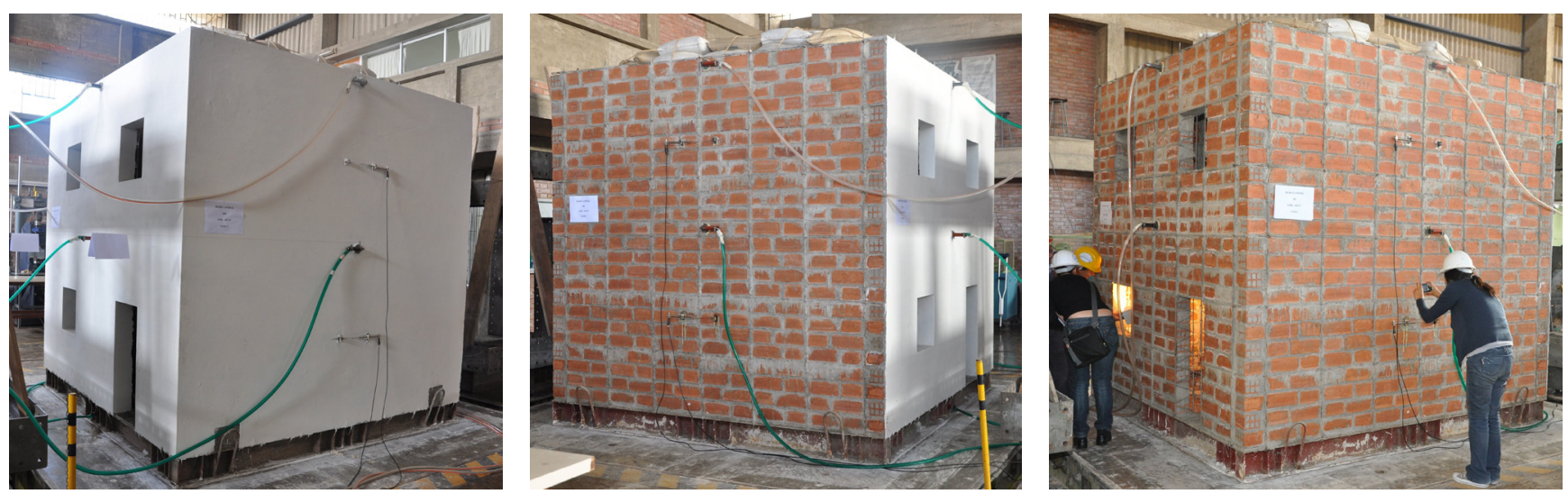

Figura 9. Prototipo 2, de ladrillo, después del ensayo sin apenas fisuras. 

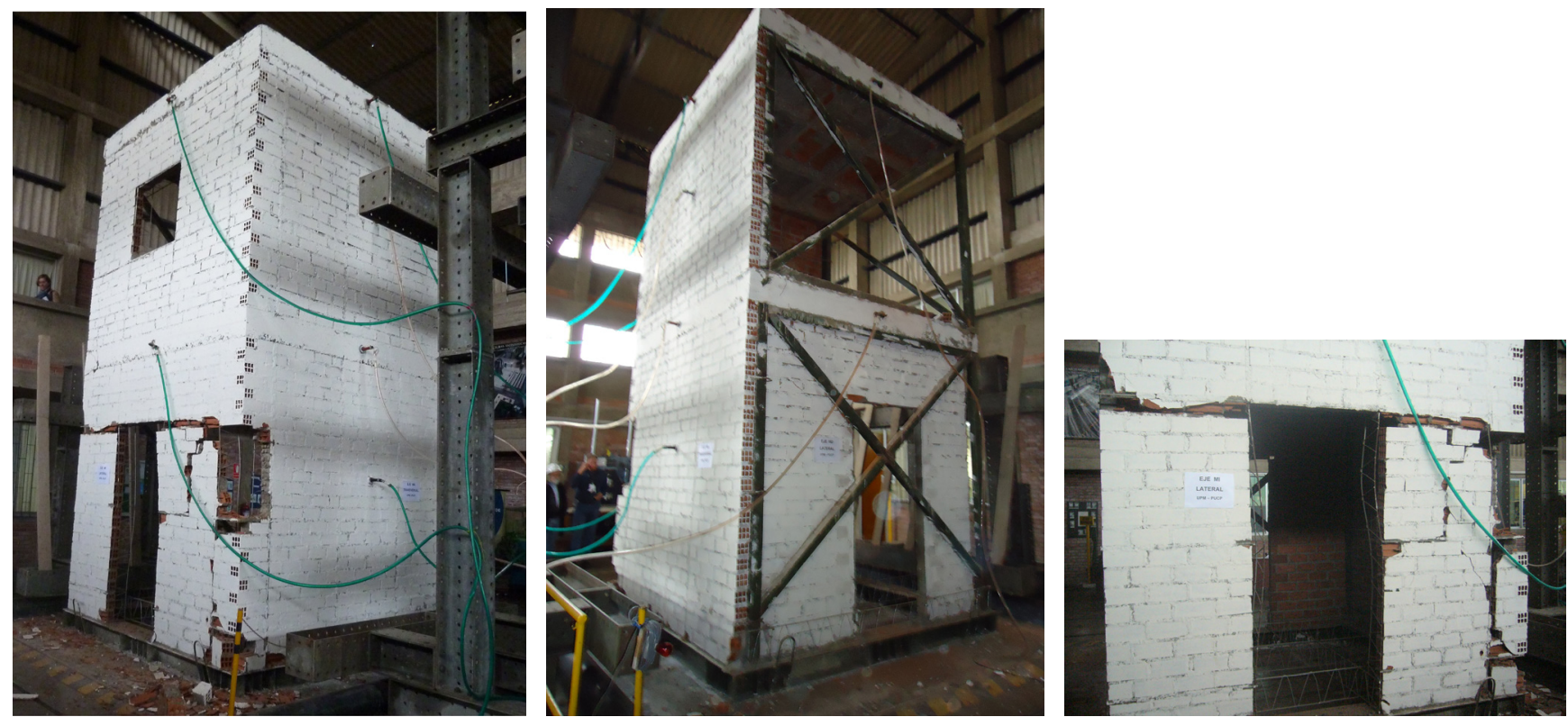

Figura 10. Prototipo 3, de ladrillo escala real, después del ensayo.

vimiento y MA y MP son los muros anterior y posterior respectivamente perpendiculares a la dirección del movimiento.

La aceleración sísmica base (PGA) de Perú es de o,4 g en la zona 3 de mayor peligrosidad sísmica según su normativa (16), a este edificio le corresponde una aceleración de cálculo de $0,33 \mathrm{~g}$ y se ensaya con una aceleración en la base de valores hasta 1,2 g, 1,5 g y 1,2 g en los prototipos 1, 2 y 3 respectivamente, por lo que los ensayos cubren con creces los valores de la norma.

En todos los casos el relleno colabora en la resistencia del sismo, permanece confinado en los espacios entre cerchas impidiendo su pandeo. En los paños no aparece la típica rotura en aspa de los cerramientos de edificios de muros sometidos a sismo. Las esquinas se muestran como una zona débil en todos los prototipos que se construyeron sin el perfil de esquina, lo que demuestra su necesidad.

La eficaz conexión de los muros entre sí y con los forjados, en las diferentes direcciones, hace que la estructura se mueva solidariamente, que los muros a pesar de tener diferente rigidez se muevan en fase, que los forjados no se caigan o se despeguen de los muros. Se observa que los muros resisten bien las acciones en su plano y fuera del plano por lo que no se producen los fallos típicos de los edificios de adobe o mampostería y no es necesario elementos de relleno de mayor calidad en las esquinas.

El comportamiento histerético de los muros izquierdo de los tres prototipos (Figura 12) muestra un comportamiento sensiblemente simétrico, similar al comportamiento cíclico del acero. Carece prácticamente de efecto «pinching» (estrangulamiento en el origen) lo que indica la gran colaboración del acero, incluso a compresión, gracias al confinamiento entre el relleno. El aspecto casi caótico de estas gráficas muestran como la posible degradación de rigidez en algunos ciclos se recupera en otros y por lo tanto una adecuada disipación de la energía.

El prototipo 2 tiene deformaciones menores al prototipo 1 del orden de la mitad, el primero de hasta $10 \mathrm{~cm}$ y el segundo nunca llega a $5 \mathrm{~cm}$ y un cortante basal del orden de 5 veces menor, debido al menor peso del ladrillo hueco frente al adobe.

El prototipo 3 (Figura 12 abajo) tiene deformaciones máximas del orden de $12 \mathrm{~cm}$ y un cortante basal del orden de 20 veces más que el prototipo 2 , coherente con el mayor peso del prototipo 3 .
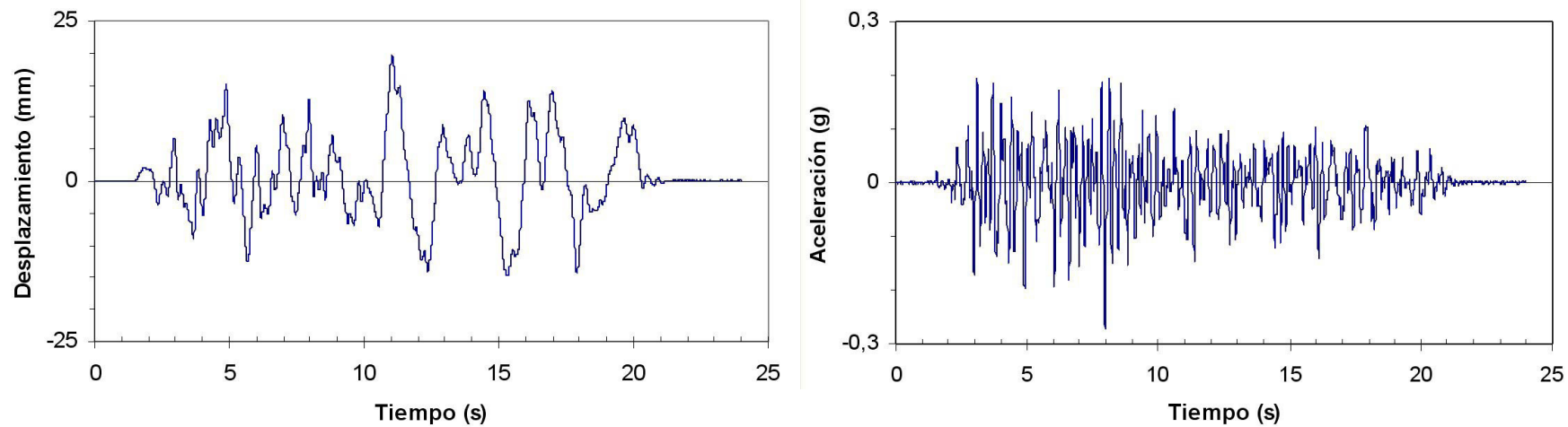

Figura 11. Movimiento de la plataforma del simulador con señal «mayo7oco» con 20 mm de desplazamiento máximo. 

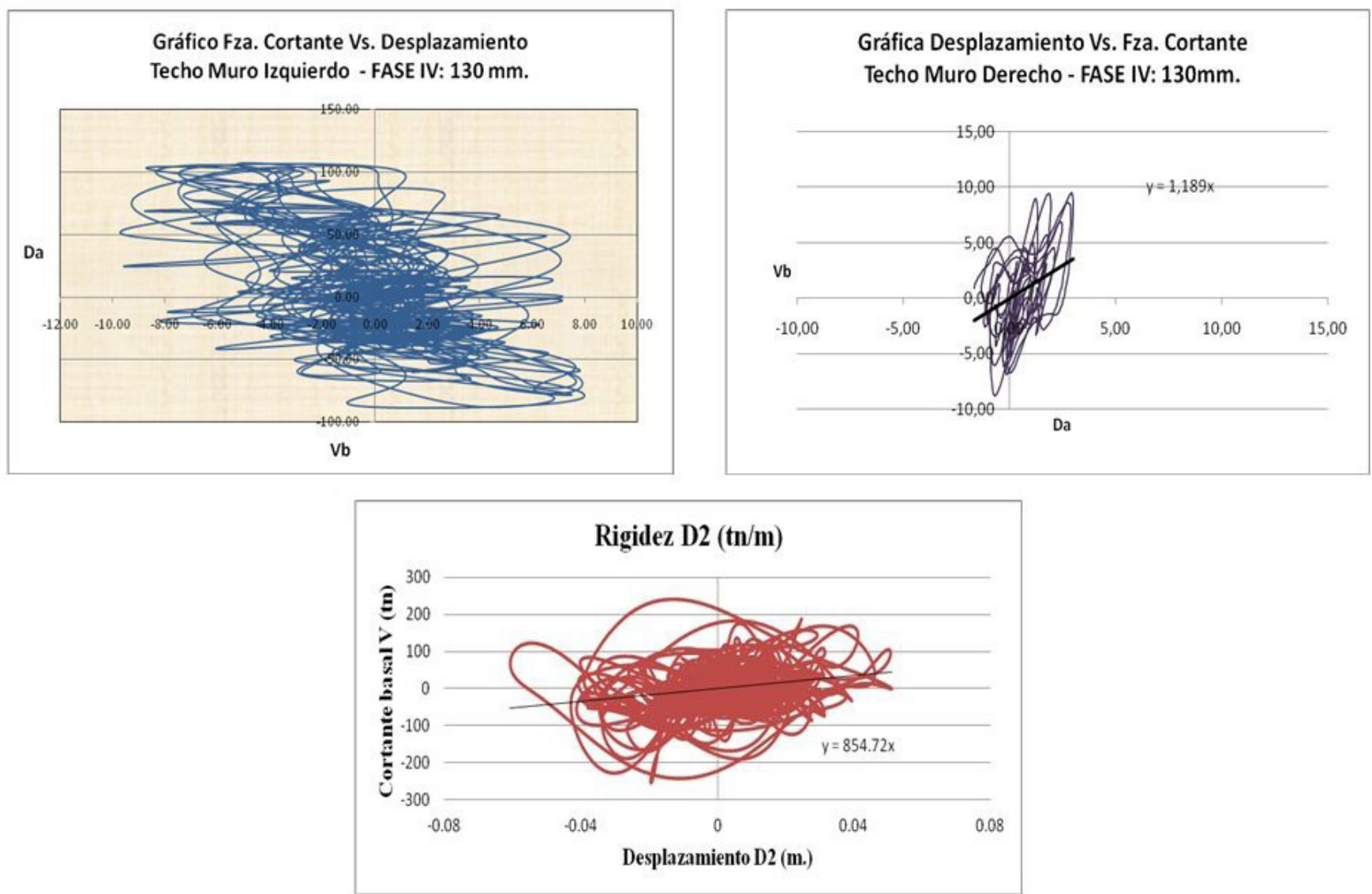

Figura 12. Comportamiento histerético de los muros izquierdo (en la dirección del desplazamiento) de prototipos 1, 2 y 3, en toneladas y cm.

\section{CONCLUSIONES}

El Sistema de Albañilería Integral, SAI, resuelve con un solo tipo de cercha de $6 \mathrm{~m}$ de longitud y con dos anchos distintos (240 $\mathrm{mm}$ y $220 \mathrm{~mm}$ ) la posibilidad de enhebrar en el espacio las armaduras, creando mallas tridimensionales para forjados y paredes que se pueden autoconstruir manualmente, dada su ligereza y simplicidad de ejecución. El secreto del SAI está en utilizar acero galvanizado preformado en cerchas que al entrelazarlas manualmente generan mallas espaciales $3 \mathrm{D}$, tanto en muros como en forjados.

Al tratarse de elementos de $6 \mathrm{~m}$ que se encuentran en sus esquinas y carecer por tanto de solapes intermedios se garantiza el trabajo en continuidad. Gracias a la longitud de las piezas se resuelve una gran variedad de tipologías de viviendas con sólo transportar un camión de $6 \mathrm{~m}$ con las armaduras. Se contempla la posibilidad de emplear cualquier material del entorno para acomodarlo entre las cerchas verticales y horizontales (tierra, adobe, ladrillo hueco, materiales reciclados procedentes de viviendas destruidas por el sismo). No obstante lo anterior, se aconseja aplicar el SAI con materiales más duraderos como bloques o prefabricados de hormigón o ladrillos huecos o perforados.

Los ensayos demuestran que el Sistema SAI resulta adecuado para la autoconstrucción de viviendas en zonas sísmicas. El sistema constructivo ha resultado de fácil puesta en práctica, a pesar de ser la primera utilización del sistema en el país, los operarios asimilaron sin mayor dificultad el procedimiento.

Los resultados de los ensayos muestran que el SAI con adobe o ladrillo tiene un comportamiento sismorresistente frente a la solicitación dinámica del simulador. La estructura permanece estable por lo que se logra el objetivo buscado. En general el sistema tiene un comportamiento altamente dúctil gracias al armado dispuesto. El prototipo 1, de adobe a escala mitad, se agrieta significativamente, el estado final es tal que, reparando las grietas provocadas se puede prolongar su vida útil y soportar sismos futuros, el prototipo 2, de ladrillo a escala mitad, tiene un comportamiento mucho mejor, sin apenas fisuras y el prototipo 3, de ladrillo a escala real, sufre grandes fisuras. El grado de daño de cada prototipo se puede clasificar de acuerdo a los grados de daño de la EMS-98 (8) para edificios de mampostería, el prototipo 1 tiene grado de daño 2, daños moderados, grietas en muchos muros (nivel de daño 2, moderado (4)), el prototipo 2, grado de daño 1, daños leves, grietas muy delgadas en muy pocos muros (nivel de daño 1 leve o despreciable (4)) y el prototipo 3, grado de daño 3, daños severos, grietas largas y extensas en casi todos los muros (nivel de daño 2, moderado (4)). En todos los prototipos los daños estructurales una vez reparados con un coste bajo puede seguir la vida útil de la estructura y siempre se protege la vida de las personas al no haber desprendimiento de materiales.

Se puede afirmar que el sistema SAI es resistente al sismo muy frecuente y poco intenso con un periodos de retorno esperado de 50 años con daños y reparaciones mínimas, en caso de realizarse con ladrillo, y compatibles con el uso simultaneo de la vivienda. Resistente al sismo frecuente con la probabilidad de ocurrir cada 200 años con daños no estructurales y de rápida reparación permitiendo la ocupación inmediata. Y resistente al sismo de proyecto con un periodo de retorno de 500 años, permitiendo la protección de las vidas, la estructura no colapsa, con daños no estructurales pero sin permitir la ocupación inmediata por necesitar la reparación del relleno y la revisión de la armadura. 


\section{REFERENCIAS}

(1) Adell, J., Bustamante, R., Dávila, D. (2005). La vivienda de adobe sismorresistente con el Sistema de Albañilería Integral. Seminario Internacional SismoAdobe. Lima, Perú.

(2) Adell, J., Lauret, B. (2005). El sistema de albañilería integral AllWall con BHH/BLOC+. Informes de la Construcción, 57(495): 33-43.

(3) Adell, J., Orta, B., Bustamante, R., Martínez, S., Orenes, B. (7-9 julio 2014). Comparison of the seismic behaviuour between three building tests, all based on a two storey model house. 9th International Masonry Conference. Guimarães, Portugal.

(4) Barbat, A. H. El riesgo sísmico en el diseño de edificios. Madrid: Ed. Calidad Siderúrgica.

(5) Blondet, M., Vargas, J., Tarque, N., Iwaki. C. (julio-septiembre 2011). Construcción sismorresistente en tierra: la gran experiencia contemporánea de la Pontificia Universidad Católica del Perú. Informes de la Construcción, 63(523): 41-50.

(6) Bustamante, R., et al. (2012). Innovación tecnológica de viviendas sismorresistentes de adobe armado con cerchas de acero galvanizado. IX Conferencia Internacional sobre el Estudio y Conservación del Patrimonio Arquitectónico de Tierra.

(7) Figueiredo, A., Varum, H., Costa, A., Silveira, D., Oliveira, C. (2013). Seismic retrofitting solution of an adobe masonry wall. Materials and Structures, 46: 213-219.

(8) Grünthal, G. (editor) (1998). Escala Macrosísmica Europea 1998. Cahiers du Centre Européen de Géodynamique et de Séismologie.

(9) Lang, A. F., Marshall, J. D. (2011). Devil in the Details: Success and Failure of Haiti's Nonengineered Structures. Earthquake Spectra, October 2011, 27(S1): S345-S372. doi: http://dx.doi.org/10.1193/1.3638136.

(10) Miyamoto, H. K., Gilani, A. S. J., Wong, K. (2011). Massive Damage Assessment Program and Repair and Reconstruction Strategy in the Aftermath of the 2010 Haiti Earthquake. Earthquake Spectra, October 2011, 27(S1): S219-S237. doi: http://dx.doi.org/10.1193/1.3631293.

(11) Orta, B., Adell, J., Bustamante, R., García-Santos, A. (2009). Ensayo en Lima (Perú) de edificio de adobe sismorresistente construido con el sistema de albañilería integral. Informes de la Construcción, 61(515): 59-65. doi: http://dx.doi. org/10.3989/ic.09.006.

(12) Orta, B., et al. (enero-marzo 2012). Experimental study of the Integral Masonry System in the construction of earthquake resistant houses. Materiales de Construcción, 62(305): 67-77. doi: http://dx.doi.org/10.3989/mc.2012.63810.

(13) Vargas, J., Bariola, J., Blondet, M., Kehta, P. K. (july-august 1986). Seismic Stregth of adobe Masonry. Materials and Structures, 19(4): 253-258.

(14) Vanin, A., Foraboschi, P. (2012). In-plane behavior of perforated brick masonry walls. Materials and Structures, 45: 1019-1034.

(15) Varum, H., Figueiredo, A., Silveira, D., Martins, T., Costa, A. (julio-septiembre 2011). Investigaciones realizadas en la Universidad de Aveiro sobre caracterización mecánica de las construcciones existentes en adobe en Portugal y propuestas de rehabilitación y refuerzo. Resultados alcanzados. Informes de la Construcción, 63(523): 127-142.

(16) Ministerio de Vivienda, Construcción y Saneamiento. (2003). NTE E.o3o Diseño Sismorresistente. Lima, Perú.

(17) TISESISMOADOBE (2008). Canal Youtube de Tisesismoadobe: Adobe sismorresistente fase 4 del ensayo del prototipo 1/2 en 2008. http://www.youtube.com/watch?v=xZ5QtM3PNNc.

(18) TISESISMOADOBE (2009). Canal Youtube de Tisesismoadobe: Construcción sismorresistente de Ladrillo. Fase 4 del ensayo del prototipo 1/2 en 2009. http://www.youtube.com/watch?v=kyhBoRdAt54. 